\title{
Fish fillet authentication by image analysis
}

\author{
Silvia Grassi ${ }^{*}$, Ernestina Casiraghi, Cristina Alamprese \\ Department of Food, Environmental and Nutritional Sciences (DeFENS), Università degli Studi di Milano, Via Celoria, 2, 20133, Milano, Italy
}

\section{A R T I C L E I N F O}

\section{Article history:}

Available online $\mathrm{xxx}$

Keywords:

Authentication

Cod

Haddock

Grey Level Co-occurrence Matrix analysis

Linear Discriminant Analysis

\begin{abstract}
A B S T R A C T
The work aims at developing an image analysis procedure able to distinguish high value fillets of Atlantic cod (Gadus morhua) from those of haddock (Melanogrammus aeglefinus). The images of fresh G. morhua $(\mathrm{n}=90)$ and $M$. aeglefinus $(\mathrm{n}=91)$ fillets were collected by a flatbed scanner and processed at different levels. Both untreated and edge-based segmented (Canny algorithm) regions of interest were submitted to surface texture evaluation by Grey Level Co-occurrence Matrix analysis. Twelve surface texture variables selected by Principal Component Analysis or by SELECT algorithm were then used to develop Linear Discriminant Analysis models. An average correct classification rate ranging from 86.05 to $92.31 \%$ was obtained in prediction, irrespective the use of raw or segmented images. These findings pave the way for a simple machine vision system to be implemented along fish market chain, in order to provide stakeholders with a simple, rapid and cost-effective system useful in fighting commercial frauds.
\end{abstract}

\section{Introduction}

A large number of computer vision systems have been investigated and applied in the agri-food system answering the need of simple, rapid, and non-destructive but reliable evaluation tools for the assessment of food quality and safety during production and processing (Ma et al., 2016). The computer vision bases go back to '60s (Baxes, 1994), though its implementation in the food industry grew mainly in the last two decades. Even if food products are extremely different, computer vision is a cross-approach aiming at the estimation of color, morphological features and surface texture characteristics directly linked to food quality and safety.

Few vision systems have been applied in the fishery industry, as reported in the reviews by Mathiassen et al. (2011), Dowlati et al. (2012), and Zion (2012). Main applications are devoted to fish counting, definition of several physical parameters (e.g. length, width, thickness, volume, weight, perimeter, area, compactness and roundness) (Balaban and Ayvaz, 2016), gender identification, chemical, biochemical and sensory quality assessment, as well as species and stock identification (Mathiassen et al., 2011). They have been implemented both in aquaculture or fish farm and in industrial conveyor belts during processing operations.

Promising results have been achieved in species identification. Zion et al. (1999) were able to correctly classify grey mullet images acquired under different lighting conditions. Storbeck and Daan (2001) described a system to recognize fish species by computer vision and a neural network, reaching more than $95 \%$ of correctly classified fish. White et al. (2006) implemented a computer vision machine to identify and measure different species with an accuracy

\footnotetext{
* Corresponding author.

Email address: silvia.grassi@unimi.it (S. Grassi)
}

ranging from 95.8 to $98.8 \%$. Alsmadi et al. (2011) extracted several features, based on ventral part of fish images, for the differentiation between fish families, especially between poison and non-poison families. All these systems are more or less complex and implemented at different levels of the fish market chain, but they all deal with intact whole fishes. To the best of our knowledge, no systems have been thought for species identification in fish fillets. However, the actual food market, driven by consumers' needs for healthy but ready-to-cook products, highly demands for fillets more than whole fishes. Thus, rapid and easy tools for authenticity assessment of these products are needed to face economic frauds (e.g. the substitution of valuable species with cheaper ones) along the whole fish supply chain.

Even if there are a number of recognized techniques for food authentication, such as molecular, chromatographic, and isotopic techniques, genomics, proteomics, vibrational and fluorescence spectroscopy, NMR and non-chromatographic mass spectrometry (Danezis et al., 2016), portable technologies for rapid and non-destructive testing would be advantageous (Stadler et al., 2016). In particular, vision systems could respond to the need of fast, reliable, non-destructive, and in situ analyses for fish authentication.

A fundamental role in computer vision is played by image analysis, which is composed by three main steps: low-level processing (i.e. image acquisition and pre-processing), intermediate-level processing (i.e. segmentation and object measurement), and advanced image processing. All these steps should be optimized in order to meet the defined purpose of the developed computer vision system, while reducing potential errors and ensuring result accuracy (Brosnan and Sun, 2004). Thus, the aim of this work was the development of an image analysis procedure to be implemented in a vision system in order to distinguish high value fillets of Atlantic cod (Gadus morhua) from those of haddock (Melanogrammus aeglefinus). 


\section{Materials and methods}

\subsection{Samples}

Fresh Gadus morhua (Gm, $\mathrm{n}=90)$ and Melanogrammus aeglefinus (Ma, $\mathrm{n}=91$ ) were provided by a trusted supplier (Copromar S.r.1., Milan, Italy) in thirteen different batches from March to June 2016. The left fillets were portioned from the whole fishes by qualified personnel and carried to the university laboratories ensuring the cold chain. Samples were stored at $4 \pm 1{ }^{\circ} \mathrm{C}$ prior to analyses that were performed within the sampling day.

\subsection{Fillet morphological characterization}

The method proposed by Malandra and Baldisserotto (2014) was applied to characterize the collected fillets according to their mor- phology. It is focused on some characteristics of the external side of the fillets, fundamental to distinguish G. morhua and M. aeglefinus. In particular, the analysis of myomeres and myosepta organization and orientation in the cranial region of the external side permitted to discriminate the considered species. Indeed the myosepta are angled against the line of the body with the innermost edge nearer the front of the body and the outermost edge nearer the tail, thus shaping like a "W". The characteristic "W" defines three main angles, one for each change of direction, called dorsal posterior (DP), central anterior (CA) and ventral posterior (VP). In the cranial region of G. morhua fillets, the W-shaped myomeres have small angle amplitude, symmetry in DP and VP angles and DP angle touching the dorsal side by an imaginary line perpendicular to the lateral line (Fig. 1a). In the cranial region of M. aeglefinus fillets (Fig. 1b), the W-shaped segments have angles broader than those in G. morhua and the imaginary line touches both the PD angle, but in the front side, and the VP angle close to the lateral line.
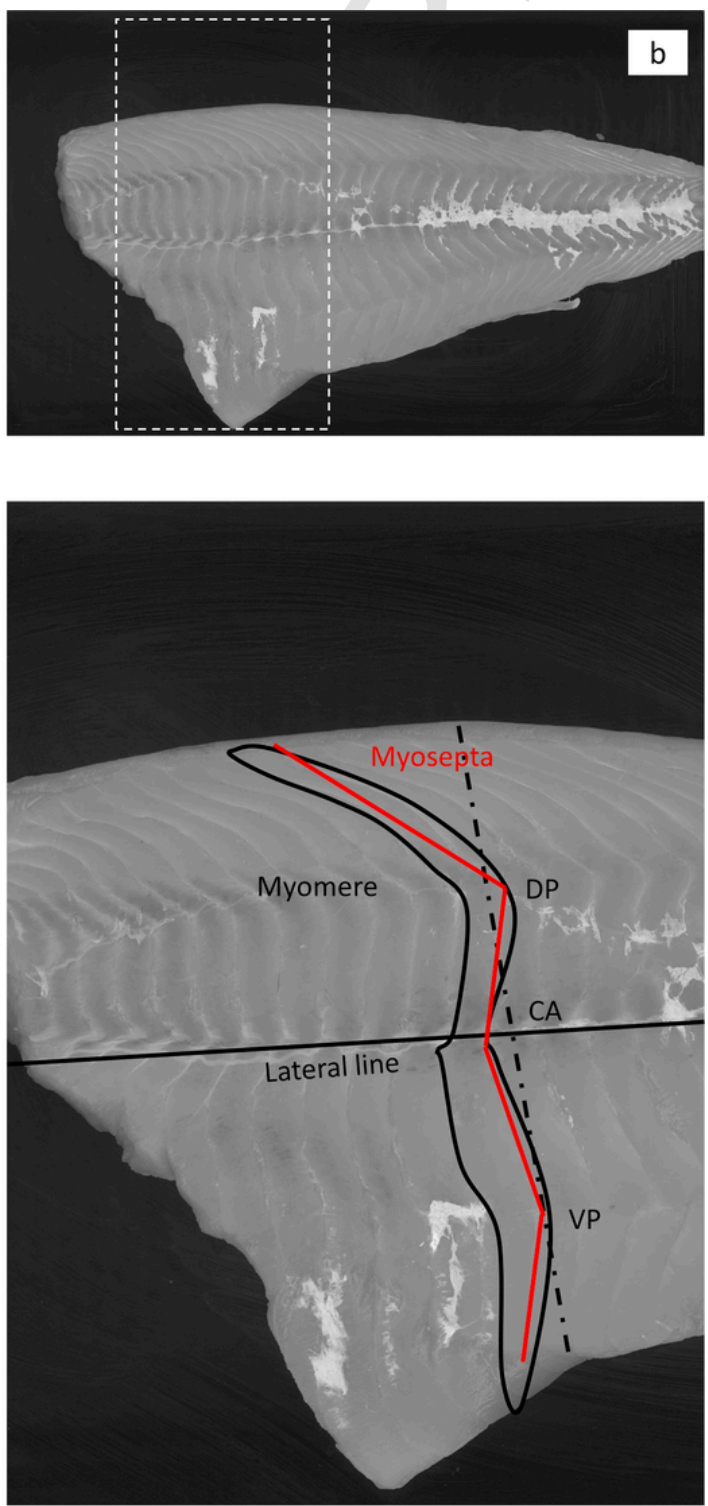

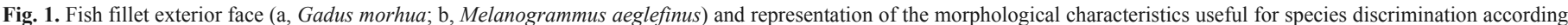

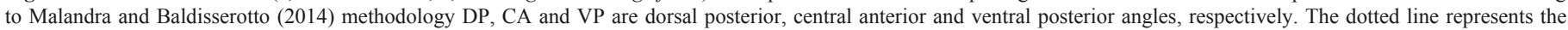
imaginary line proposed by Malandra and Baldisserotto (2014) to evaluate the W-shape of myosepta. 


\subsection{Image analysis}

\subsubsection{Image low-level processing}

The acquisition of the images of each fillet was performed with a flatbed scanner (HP Scanjet 8300, HP Inc., Palo Alto, CA, USA), covering samples with a black box to prevent light losses. Images were acquired at a resolution of $600 \mathrm{dpi}$, with a color depth of 48 bit and saved in uncompressed TIFF format. Image analysis was carried out on a selected region of interest (ROI; $800 \times 1200$ pixels) cropped in the cranial area of each fillet and converted in grayscale ( 8 bit).

\subsubsection{Image intermediate-level processing}

Each image ROI was edge-based segmented through Canny multi-stage algorithm. The application of this algorithm aimed at significantly reducing the amount of data by filtering useless information out while preserving the important structural properties in the image, represented in this case by the muscular tissue pattern. In details, Canny edge algorithm consists of: noise reduction by Gaussian filter; image intensity gradient identification with four filters to detect horizontal, vertical and diagonal edges in the blurred image; non-maximum suppression to define a set of edge points known as "thin edges"; edges' tracing through a double thresholding; suppression of all the edges that are weak and not connected to strong edges by hysteresis (Nosrati et al., 2013).

\subsubsection{Image high-level processing}

Both untreated and edge-based segmented ROI matrices were submitted to high level processing, through surface texture evaluation by Grey Level Co-occurrence Matrix (GLCM) analysis and multivariate analysis.

GLCM, a classical second-order statistical method, was applied to create a symmetric matrix reporting the frequency of the different combinations of grey levels co-occurring in the selected ROIs. Indeed, it calculates how often two pixels with intensity values $i$ and $j\left(p_{i, j}\right)$ at a particular distance $(\delta)$ along a given direction (expressed in angles, $\theta$ ) occur in an image. Since the calculation is strongly affected by pixel pitch and direction, a single GLCM might not be enough to describe textural features of the input image. For this reason, 40 GLCMs for a single input image were calculated considering ten distances $(\delta$ from 1 to 10 pixels $)$ and all the four directions $\left(\theta\right.$ of $\left.0^{\circ}, 45^{\circ}, 90^{\circ}, 135^{\circ}\right)$. Then, four main texture features were calculated for each matrix. Texture feature calculation uses the GLCM to give a measure of the intensity variation among the pixels of interest (Haralick et al., 1973). In this work, the following features were calculated:

- Contrast: it measures the intensity contrast between a pixel and its neighbor over the whole image, evaluating the local variation

$$
\text { Contrast }=\sum_{i} \sum_{j}(i-j)^{2} p_{i j}
$$

It ranges from 0 (for a constant image) to the root mean square of the size of GLCM-1.

- Correlation: it measures how a pixel is correlated to its neighbor over the whole image, evaluating the joint probability occurrence of specified pixel pairs

$$
\text { Correlation }=\sum_{i} \sum_{j} \frac{(i-\mu i)(j-\mu j)}{\sqrt{\left(\sigma i^{2}\right)\left(\sigma j^{2}\right)}} p_{i j}
$$

where $\mu$ and $\sigma$ are mean and standard deviation values, respectively. It ranges between -1 and 1 , which stand for a perfectly negatively or positively correlated image.

- Energy, also known as uniformity or angular second moment: it returns the sum of squared elements in the GLCM

$$
\text { Energy }=\sum_{i} \sum_{j} p_{i j}^{2}
$$

It ranges from 0 to 1 , being 1 the value for a constant image.

- Homogeneity, or Inverse Difference Moment: it measures the closeness of the distribution of elements in the GLCM to the GLCM diagonal

$$
\text { Homogeneity }=\sum_{i} \sum_{j} \frac{1}{1+(i-j)^{2}} p_{i j}
$$

It ranges from 0 to 1 . Homogeneity is 1 for a diagonal GLCM.

Considering these four texture features, two matrices composed of 181 samples and 160 variables were obtained for the untreated and the edge-based segmented data. Multivariate analysis was then carried out by Principal Component Analysis (PCA) followed by Linear Discriminant Analysis (LDA). PCA was carried out by Matlab R2016a software (The MathWorks Inc., USA) on the autoscaled matrices to investigate sample distribution and variable weights for feature selection. Variable selection was also performed by SELECT algorithm, implemented in V-PARVUS package (Forina et al., 2008), which searches for the variable with the largest Fisher weight, selects it decorrelating then the other predictors, and iterates until a fixed number of variables is chosen. The reduced datasets obtained were submitted to LDA (Forina et al., 2007) using the V-PARVUS package. Each sample of fish fillets was assigned to one of the two a-priori classes defined based on the belonging species (Gm for Gadus morhua and Ma for Melanogrammus aeglefinus).

Classification models were calibrated with $70 \%$ of the collected samples (121 samples) validated both in cross validation (with 5 cancellation groups) and in prediction with three different external test sets each representing 4 out of the 13 different sampling batches (i.e. $30 \%$ of the collected images with balanced representation of the two fish species).

\section{Results and discussion}

\subsection{Fillet morphological characterization}

Visual inspection of the muscular tissue structure was carried out for the collected samples by means of the procedure proposed by Malandra and Baldisserotto (2014). Each examined fillet resulted to belong to the claimed species. However, even though the applied methodology confirmed to be reliable, it is not difficult to imagine how complex the recognition of differences in myomeres and myosepta fibers' orientation by visual perception could be and how long the effective screening of each fillet could take. Moreover, very well trained personnel is needed. 


\subsection{Image intermediate-level processing}

Fig. 2a and $\mathrm{b}$ report an example of ROIs obtained from $\mathrm{Gm}$ and Ma samples. A visual interpretation based on the W-shape of muscular fibers is quite difficult and long to carry out. Thus, the ROIs were submitted to segmentation by Canny method, in order to identify and locate sharp discontinuities (Fig. 2c and d). The Canny algorithm was optimized as follows: ROIs were treated with a Gaussian filter with an intermediate width (4) to detect and remove with good sensitivity the noise, while keeping a low localization error of Canny algorithm. The following step dealt with finding the intensity gradient of the image in four directions (vertical, horizontal and the two diagonals), in order to highlight regions with high spatial derivatives. Successively, non-maximum suppression was applied to trace and suppress any pixel value that is not considered an edge (i.e. which has an intensity equal to zero). The result was a gradient array to be further reduced by thresholding. Canny thresholding, based on hysteresis, requires two constraints: an intensity of 0.4 was used in the present study as higher threshold to mark out the remarkable edges; a lower threshold (0.08) was applied to trace faint sections of edges. This approach gave good results in defining edges along continuous curves in the image, thus it made possible to follow a faint section of a given line and to discard a few noisy pixels with large gradient but not constituting the actual line (Nosrati et al., 2013). In the obtained binary images (Fig. 2c and d) each edge pixel is drawn in white and it is evident that the Canny algorithm helped in enhancing fish muscular structure, even if it did not extract only the myosepta information. However, it performed better than other edge-based segmentation approaches (Laplacian of Gaussian, Sobel, Prewitt and Robert's operator), confirming a low error rate if compared to other techniques (Maini and Aggarwal, 2009).

\subsection{Image surface texture features}

The complete results of surface texture analysis by GLCM cannot be shown due to the big size of the datasets (181 samples $\times 160$ variables). The main findings are summarized in Fig. 3, which reports, for the untreated and edge-based segmented data of the two fish species, the average values of the four calculated features (i.e. contrast, correlation, energy and homogeneity) for the ten considered distances (from 1 to 10 pixels) and the four angles $\left(0^{\circ}, 45^{\circ}, 90^{\circ}\right.$ and $\left.135^{\circ}\right)$.

Surface texture features of the untreated data (Fig. 3a) showed no differences between the two fish species for contrast and homogeneity that are, actually, inversely correlated. Contrast gives a description of the intensity contrast between a pixel and its neighbor over the whole image. In our analyses it could assume values from 0 to 49 (i.e. $\left.(8-1)^{\wedge} 2\right)$, but the average values calculated for $\mathrm{Gm}$ and Ma fillets were $0.11 \pm 0.05$ and $0.10 \pm 0.06$, respectively. Thus, the images can be considered constant at any tested distance and angle. Homogeneity (also known as Inverse Difference Moment) values ranged from 0.89 to 0.97 , being close to the diagonal GLCM that has, by definition, a value of 1 . These results revealed that there was a small grey tone difference in the pixel pair comparison of the fish fillet images. The smaller the pixel distance, the higher the homogeneity, meaning that at $\delta=1$ the GLCM is diagonal, i.e. all elements at local level in the image are the same. The contrast and homogeneity results obtained were expected since, as it can be observed by the examples reported in Fig. $2 \mathrm{a}$ and $\mathrm{b}$, the fish fillet images had very low contrast, with a high evenness. On the other hand, a difference between the two fish species was observed based on correlation and energy values, no matter the considered distance nor direction (Fig. 3a). Correlation measures grey tone linear dependencies in the image, thus values close to 1 stand for positive correlation. Gm samples showed an average pixel correlation higher then Ma samples $(0.8 \pm 0.2$ vs. $0.7 \pm 0.2)$. An opposite trend was observed for energy, with Ma average values $(0.6 \pm 0.2)$ higher than those of $\mathrm{Gm}(0.5 \pm 0.2)$, meaning that the grey level distribution in $\mathrm{Ma}$ had a more constant or periodic form then in Gm.

Surface texture features calculated on the edge-based segmented ROIs did not show species differences (Fig. 3b) as there was a sharp overlapping of the average values.

The comparison of surface texture features calculated on the untreated and the edge-based segmented ROIs showed that data transformation particularly affected correlation and energy values; indeed the correlation highly decreased in magnitude after Canny segmenta-
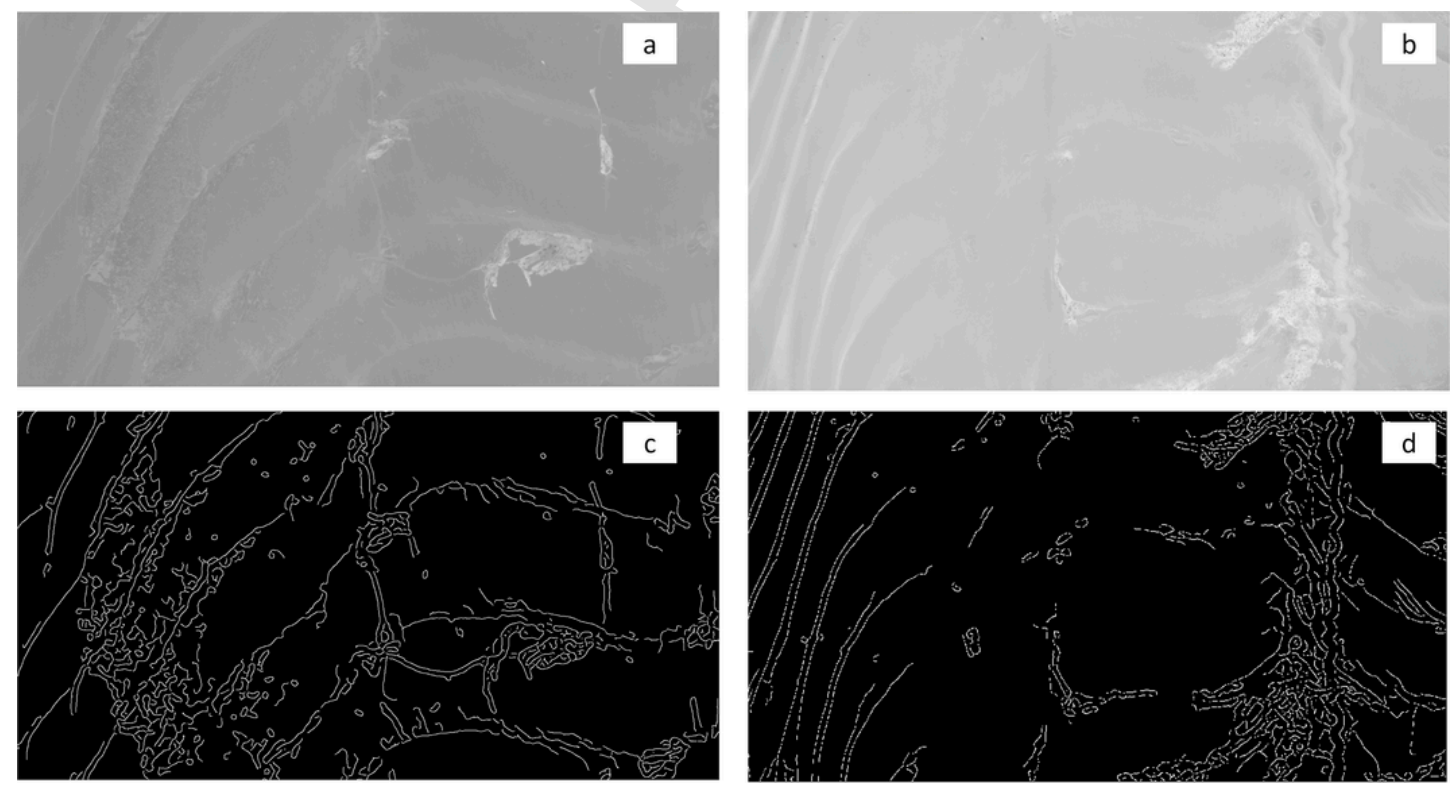

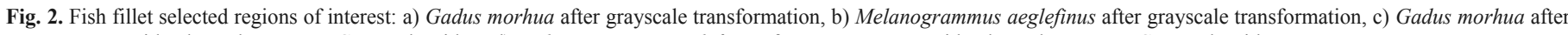
pre-treatment with edge enhancement Canny algorithm, d) Melanogrammus aeglefinus after pre-treatment with edge enhancement Canny algorithm. 
Journal of Food Engineering xxx (2018) xxx-xxx

5

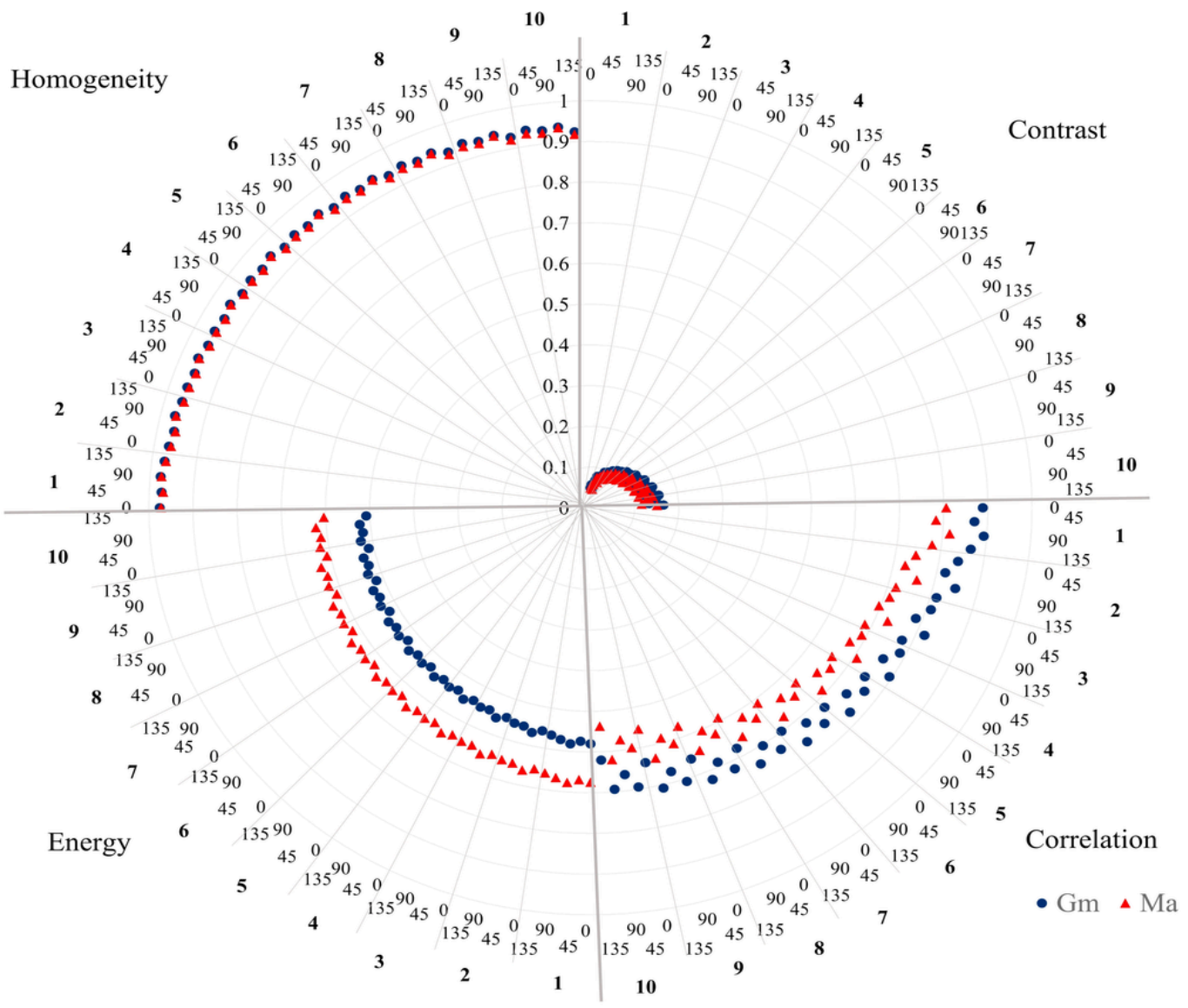

a

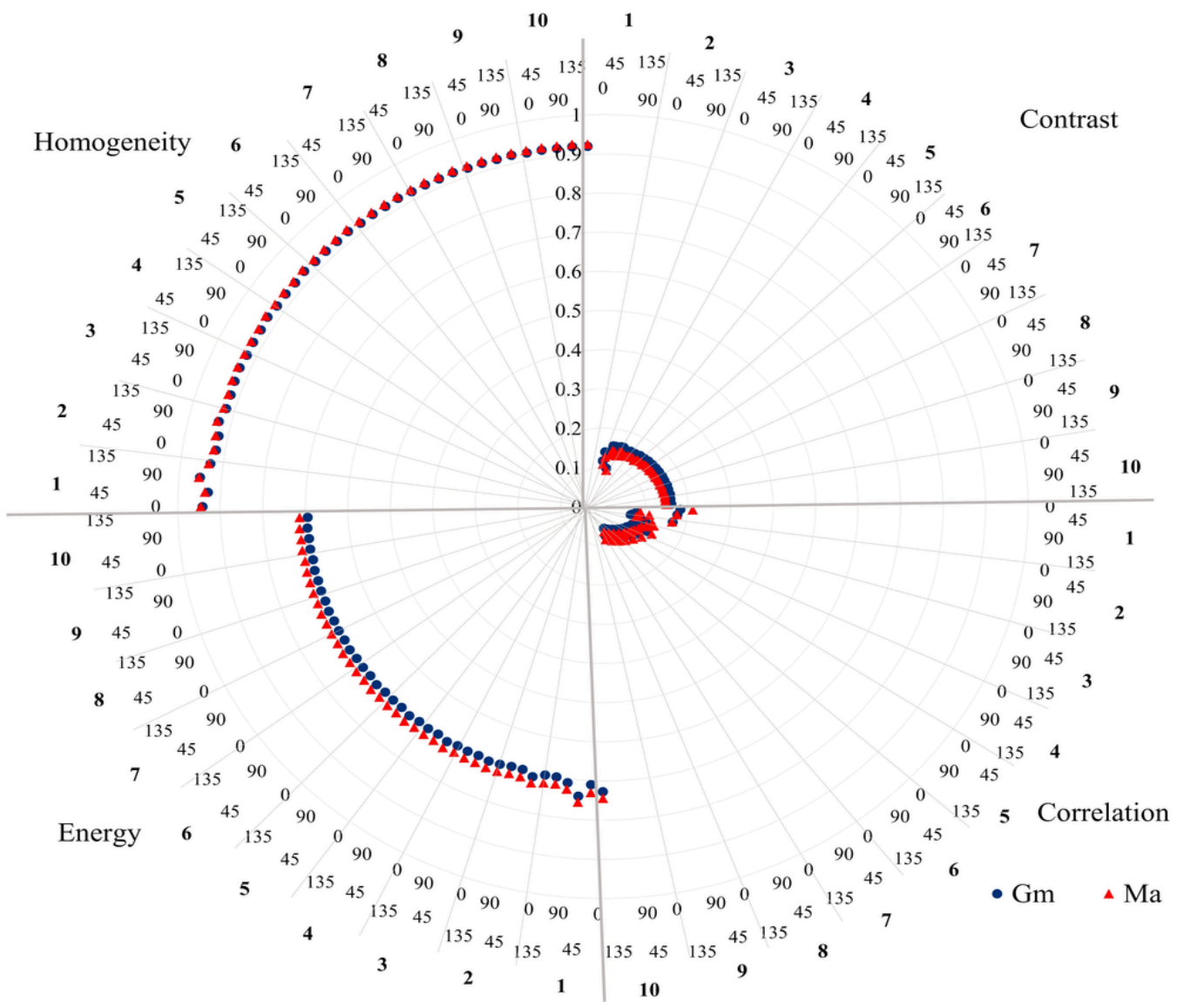

b 
tion (averaged values from 0.73 to 0.11 ), whereas energy increased (averaged value from 0.57 to 0.71 ). In any case, the two features calculated on raw or the edge-based transformed data decreased when the windows size increased.

From these observations, it seems plausible a fish species discrimination according to average surface texture features calculated with untreated data. However, in order to confirm this hypothesis, a deeper evaluation of surface texture parameters of all samples was necessary.

\subsection{Principal Component Analysis (PCA)}

PCA was applied to explore both the complete surface texture datasets (i.e. untreated and edge-based segmented data) in order to highlight possible groups of samples according to fish species and to highpoint the variables' weight in this distribution. As regards the untreated matrix (181 samples $\times 160$ variables), the bi-plot reported in Fig. 4a shows the projection of samples and variables (eigenvectors) in the space defined by PC1 and PC2 that together explained $95.43 \%$ of the total variance. No sample distribution according to fish species was evident. The bi-plot showed that each surface texture feature (i.e. contrast, correlation, energy and homogeneity) is positioned in a different quadrant, no matter the distances and the angles investigated. This means that values of a given surface texture parameter calculated with different distances and/or directions gave similar information about fish fillet samples. Homogeneity and energy are quite close to each other, suggesting a similar contribution to the sample variability on the first PC.

PCA was applied also to surface texture data calculated after edge-based segmentation of fish fillet images. Results are reported in the bi-plot of Fig. 4b, which shows the variance space of PC1 and PC2 describing $94.42 \%$ total variance. In this case too, no sample distribution was observable according to fish species, and the surface texture variables calculated at the different distances and angles were generally grouped according to the feature, with the exception of four correlation data calculated at $90^{\circ}$ that are positioned in the quadrant IV instead of quadrant I.

Because of the high correlation observed for each surface texture variable calculated at different distances and angles, a variable reduction was applied for the subsequent chemometric analyses. In particular, for each considered features, the values calculated in the four directions at a given distance were averaged, thus eliminating the effect of angles on the results (Haralick et al., 1973). As regards the distance, since no information are available on the optimal value to be considered, the two extreme gaps ( 1 and 10 pixels) and a central point (5 pixels) were used. As a result, a so-called PCA-reduced dataset composed of 181 samples and 12 variables was obtained for both untreated and edge-based segmented fish fillet images.

\subsection{Linear Discriminant Analysis (LDA)}

LDA was applied to the PCA-reduced datasets (181 samples $\times 12$ variables) obtained by GLCM analysis of untreated and edge-based segmented fish fillet ROIs. LDA permits to classify objects according to categories by the construction of the optimal a-posteriori classification rule based on a class a-priori assigned to each sample. The classification rule is quite simple as it originates from directions, or canonical variables, describing the maximum distance among cate- gories, improving the class separability (Forina et al., 2007). The correct classification rate is an index of goodness of the constructed rule but it is not sufficient to test the model reliability. Therefore, LDA models were validated by both internal cross-validation and prediction, considering three different external test sets. The correct classification percentages in prediction obtained with surface texture parameters calculated on untreated fillet images ranged from 67.44 to 76.00 depending on the external test set considered (Table 1). Similar results were obtained also with surface texture features of the edge-based segmented images, being the correct classification range 67.44-72.07\%.

Model performances were promising but not exhaustive to confirm the fish fillet species. Indeed, a stakeholder applying such approach could confuse up to $30 \%$ of the investigated samples, with the risk of accepting a $M$. aeglefinus fillet as the more valuable G. morhua one. Therefore, LDA was performed also on two other datasets constructed with the surface texture variables selected by SELECT algorithm (Forina et al., 2008). In particular, twelve features were picked up based on their Fisher classification weight (Table 2). They were mainly related to contrast when considering the untreated matrices; indeed 7 out of 12 variables referred to this feature calculated at different distances and angles. Contrast was a significant parameter maybe because it is specifically related to surface texture patterns of an image (Haralick et al., 1973). The variables selected for the pre-treated dataset were instead almost exclusively related to correlation calculated at low distances. Indeed, a high segmentation effect on this parameter was also observed in $\S 3.3$.

Using the SELECT-reduced datasets for LDA with the same constraints previously described, reliable models were obtained with all the external test sets and for both untreated and edge-based segmented images. Indeed, the average prediction ability ranged from 86.05 to $92.31 \%$ (Table 3 ), irrespective the use of raw or segmented images.

The obtained results are in agreement with those reported in image analysis studies performed for fish species discrimination in the last 20 years. Some studies reported better classification abilities, but they were carried out on whole fishes rather than fillets, thus taking advantage of skin color, fish shape and other geometric features. Indeed, Zion et al. $(1999,2012)$ found correct classification rate in prediction from 86 to $100 \%$ when discriminating between carp, St. Peter's fish and grey mullet. In another study dealing with six different fish species (sole, plaice, whiting, dab, cod, and lemon sole), a correct classification higher than 95\% was obtained by Artificial Neural Network system (Storbeck and Daan, 2001). White et al. (2006) reached 100\% accuracy of differentiation giving an indisputable discrimination between flatfish and roundfish.

Even if still good, performances of classification models decreases when dealing with fish fillets and in any case no studies have been published so far about fish fillet authentication by means of image analysis techniques. For instance, Misimi et al. (2008) reached a 90\% correct classification rate for the rating of Atlantic cod and Atlantic salmon during rigor mortis and ice storage. Also Xu et al. (2017) applied a computer vision system for differentiation of organically and conventionally farmed salmon reaching a correct classification rate in prediction of $83.6 \%$ based on $\mathrm{L}^{*} \mathrm{a} * \mathrm{~b}$ values (PLS-DA model). Better results have been found when applying Vis/NIR imaging (Zhu et al., 2013) for discriminating fresh from frozen-thawed fillets (correct classification of $97 \%$ ), or when combining different in-

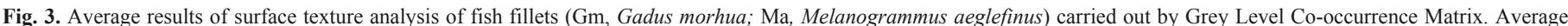

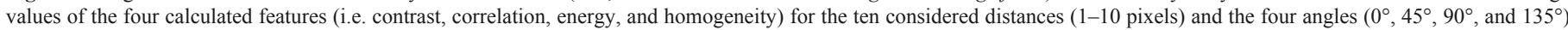
are shown: a) data obtained by untreated images, b) data obtained by images pre-treated with edge enhancement Canny algorithm. 

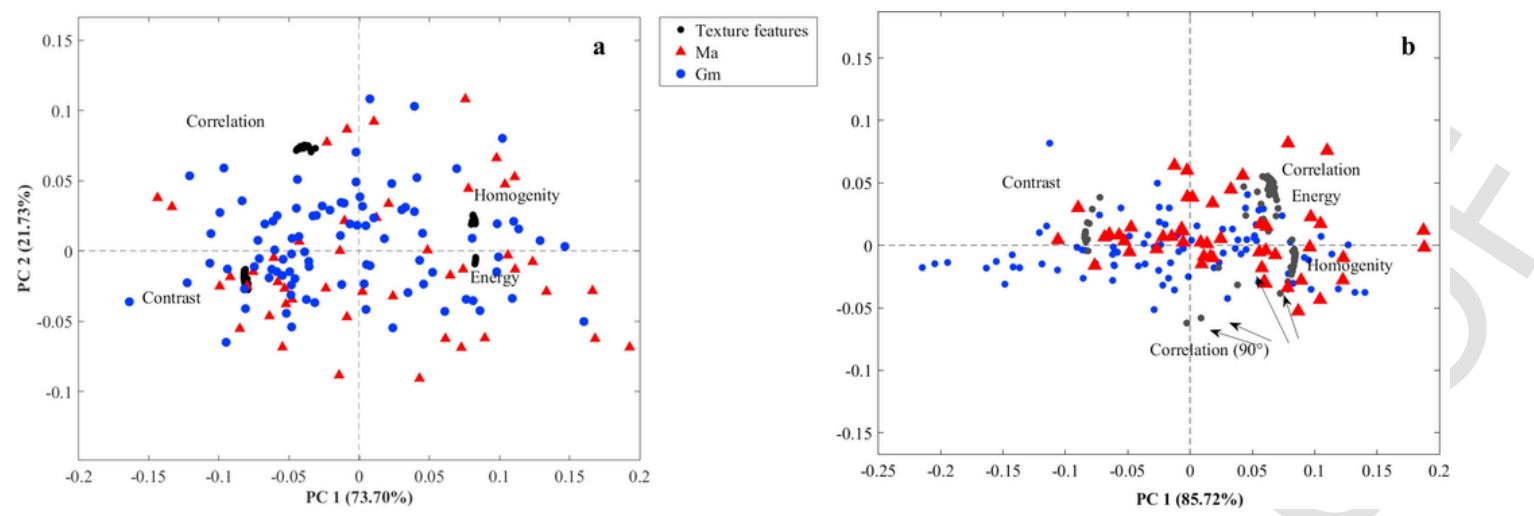

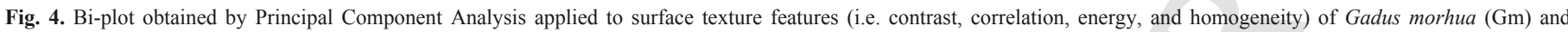
Melanogrammus aeglefinus (Ma) fillets calculated on untreated images (a) and images pre-treated with edge enhancement Canny algorithm (b).

Table 1

Results of Linear Discriminant Analysis applied for fish species authentication (Gadus morhua and Melanogrammus aeglefinus) to fillet surface texture features selected by Principal Component Analysis: average correct classification percentages of models based on untreated or edge-based segmented images.

\begin{tabular}{lll}
\hline & Untreated dataset & Pre-treated dataset \\
\hline External test set $\mathbf{1}$ & & \\
$\quad$ Calibration & 79.54 & 74.32 \\
Cross validation & 72.73 & 65.72 \\
Prediction & 73.08 & 72.07 \\
External test set 2 & & \\
$\quad$ Calibration & 85.33 & 75.78 \\
Cross validation & 78.89 & 72.22 \\
Prediction & 76.00 & 69.00 \\
External test set 3 & & \\
Calibration & 82.27 & 77.31 \\
Cross validation & 76.29 & 70.64 \\
Prediction & 67.44 & 67.44 \\
\hline
\end{tabular}

Table 2

Surface texture features of untreated or edge-based segmented images selected by SELECT algorithm.

\begin{tabular}{llllll}
\hline \multicolumn{1}{l}{ Untreated dataset } & \multicolumn{5}{l}{ Pre-treated dataset } \\
\hline Feature & Distance & Angle & Feature & Distance & Angle \\
\hline Contrast & 1 & 135 & Contrast & 1 & 45 \\
& 3 & 45 & Correlation & 1 & 0 \\
& 4 & 0 & & 2 & 0 \\
& 4 & 45 & & 2 & 45 \\
& 6 & 90 & & 3 & 135 \\
& 8 & 0 & & 4 & 0 \\
Correlation & 3 & 45 & & 4 & 45 \\
& 10 & 0 & & 4 & 135 \\
Energy & 5 & 90 & 5 & 6 & 90 \\
& 1 & 45 & & & 10 \\
& 10 & 45 & 7 & & 0 \\
& 10 & 135 & 8 & & 135 \\
\hline
\end{tabular}

struments (i.e. portable Vis/NIR spectrometer, digital camera and texture analyzer) for the discrimination of fresh and frozen-thawed West African goatfish (classification accuracy of 100\%) (Ottavian et al., 2014).

As regards cod and haddock fillet authentication, Grassi et al. (2018) proposed a near infrared spectroscopy approach able to correctly discriminate $100 \%$ of fillets. It is thus evident that systems more complex than computer vision tools, like near infrared spectroscopy and hyperspectral imaging, can lead to higher classification
Table 3

Results of Linear Discriminant Analysis applied for species authentication (Gadus morhua and Melanogrammus aeglefinus) to fillet surface texture features selected by SELECT algorithm: average correct classification percentages of models based on untreated or edge-based segmented images.

\begin{tabular}{lll}
\hline & Untreated dataset & Pre-treated dataset \\
\hline External test set 1 & & \\
Calibration & 92.73 & 91.59 \\
Cross validation & 84.09 & 86.36 \\
Prediction & 92.31 & 92.31 \\
External test set 2 & & \\
Calibration & 92.67 & 92.22 \\
Cross validation & 86.67 & 88.89 \\
Prediction & 90.00 & 92.00 \\
External test set 3 & & \\
Calibration & 92.99 & 92.58 \\
Cross validation & 84.54 & 85.57 \\
Prediction & 86.05 & 86.05 \\
\hline
\end{tabular}

rate, but these instruments have higher initial capital investment and onerous data analysis if compared to the image analysis approach here proposed.

\section{Conclusions}

This work demonstrated a good performance of image analysis applied to Gadus morhua and Melanogrammus aeglefinus fillet discrimination. In particular, the application of GLCM analysis and the selection of surface texture parameters by the algorithm SELECT allowed to obtain reliable LDA models reaching correct classification rate in prediction up to $92 \%$.

These findings envision a machine vision system to be implemented from the fish factory gate, where the material is delivered, up to the retail channels. In particular, scanning windows could be set directly on conveyor sorting belts in industries producing transformed fish products, in order to check the authenticity of the raw materials. In a future perspective, also simple scanning machines could be developed in order to make them available in markets and supermarkets, allowing consumers to scan products before purchasing. Providing stakeholders with such a simple, rapid and cost-effective system will help them in fighting commercial frauds throughout the entire fish value chain.

\section{Compliance with ethical standards}

Disclosure of potential conflicts of interest. 


\section{Conflicts of interest}

The authors declare that they have no conflict of interest. Research involving Human Participants and/or Animal.

\section{Ethical approval}

This article does not contain any studies with human participants or animals performed by any of the authors.

\section{Uncited References}

Sivertsen et al., 2011.

\section{References}

Alsmadi, M.K., Omar, K.B., Noah, S.A., 2011. Fish classification based on robust features extraction from color signature using back-propagation classifier. J. Comput. Sci. 7 (1), 52-58

Balaban, M.O., Ayvaz, Z., 2016. Measurement of visual attributes of fresh and processed seafood. In: Genç, İ.Y., Esteves, E., Diler, A. (Eds.), Handbook of Seafood: Quality and Safety Maintenance and Applications. Nova Science Publishers Incorporated, New York, pp. 66-85.

Baxes, G.A., 1994. Digital Image Processing Principles and Applications. Wiley, New York

Brosnan, T., Sun, D.-W., 2004. Improving quality inspection of food products by computer vision - a review. J. Food Eng. 61, 613-616.

Danezis, G.P., Tsagkaris, A.S., Camin, F., Brusic, V., Georgiou, C.A., 2016. Food authentication: techniques, trends and emerging approaches. Trac. Trends Anal. Chem. 85, 123-132.

Dowlati, M., de la Guardia, M., Mohtasebi, S.S., 2012. Application of machine-vision techniques to fish-quality assessment. Trac. Trends Anal. Chem. 40, 168-179.

Forina, M., Lanteri, S., Casale, M., Cerrato Oliveros, M.C., 2007. Stepwise orthogonalization of predictors in classification and regression techniques: an "old" technique revisited. Chemometr. Intell. Lab. Syst. 87, 252-261.

Forina, M., Lanteri, S., Armanino, C., Casolino, C., Casale, M., Oliveri, P., 2008. V-PARVUS 2008. University of Genova http://www.parvus.unige.itAccessed 25 March 2017.

Grassi, S., Casiraghi, E., Alamprese, C., 2018. Handheld NIR device: a non-targeted approach to assess authenticity of fish fillets and patties. Food Chem. 243, $382-388$
Haralick, R.M., Shanmugam, K., Dinstein, I., 1973. Textural features for image classification. IEEE Trans. Syst. Man Cybern. 6, 610-621.

Ma, J., Sun, D.-W., Qu, J.H., Liu, D., Pu, H., Gao, W.H., Zeng, X.A., 2016. Applications of computer vision for assessing quality of agri-food products: a review of recent research advances. Crit. Rev. Food Sci. Nutr. 56 (1), 113-127.

Maini, R., Aggarwal, H., 2009. Study and comparison of various image edge detection techniques. Int. J. Image Process. 3 (1), 1-11.

Malandra, R., Baldisserotto, G., 2014. Identificazione dei filetti delle principali specie di Gadidi mediante l'osservazione della struttura muscolare. Il Pesce 2, 114-118.

Mathiassen, J.R., Misimib, E., Bondøb, M., Veliyulinb, E., Østvik, S.O., 2011. Trends in application of imaging technologies to inspection of fish and fish products. Trends Food Sci. Technol. 22, 257-275.

Misimi, E., Erikson, U., Digre, H., Skavhaug, A., Mathiassen, J.R., 2008. Computer vision-based evaluation of pre- and post-rigor changes in size and shape of Atlantic cod (Gadus morhua) and Atlantic salmon (Salmo salar) fillets during rigor mortis and ice storage: effects of perimortem handling stress. J. Food Sci. 73 (2), E57-E68.

Nosrati, M., Karimi, R., Hariri, M., Malekian, K., 2013. Edge detection techniques in processing digital images: investigation of Canny algorithm and Gabor method. World Appl. Program. 3 (3), 116-121.

Ottavian, M., Fasolato, L., Serva, L., Facco, P., Barolo, M., 2014. Data fusion for food authentication: fresh/frozen-thawed discrimination in West African Goatfish (Pseudupeneus prayensis) fillets. Food Bioprocess Technol. 7 (4), 1025-1036.

Sivertsen, A.H., Kimiya, T., Heia, K., 2011. Automatic freshness assessment of cod (Gadus morhua) fillets by Vis/Nir spectroscopy. J. Food Eng. 103, 317-323.

Stadler, R.H., Tran, L.A., Cavin, C., Zbinden, P., Konings, E.J., 2016. Analytical approaches to verify food integrity: needs and challenges. J. AOAC Int. 99 , 1135-1144.

Storbeck, F., Daan, B., 2001. Fish species recognition using computer vision and a neural network. Fish. Res. 51 (1), 11-15.

White, D.J., Svellingen, C., Strachan, N.J.C., 2006. Automated measurement of species and length of fish by computer vision. Fish. Res. 80 (2), 203-210.

Xu, J.L., Riccioli, C., Sun, D.W., 2017. Comparison of hyperspectral imaging and computer vision for automatic differentiation of organically and conventionally farmed salmon. J. Food Eng. 196, 170-182.

Zhu, F., Zhang, D., He, Y., Liu, F., Sun, D.-W., 2013. Application of visible and near infrared hyperspectral imaging to differentiate between fresh and frozen-thawed fish fillets. Food Bioprocess Technol. 6 (10), 2931-2937.

Zion, B., 2012. The use of computer vision technologies in aquaculture-a review. Comput. Electron. Agric. 88, 125-132.

Zion, B., Shklyar, A., Karplus, I., 1999. Sorting fish by computer vision. Comput. Electron. Agric. 23 (3), 175-187. 